\title{
Commitment devices in the treatment of diabetic foot ulcers
}

\author{
Gustav Jarl ${ }^{1,2}$ (D)
}

\begin{abstract}
Background: Non-removable offloading devices are recommended for the treatment of uncomplicated plantar diabetic foot ulcers because adherence to using removable devices is low. However, patients may not always understand how crucial the non-removability is to ulcer healing, leaving them with the impression that it is the device per se that heals the ulcer. Thus, after ulcer healing when patients return to using removable offloading devices, typically therapeutic footwear, they often return to a low level of adherence resulting in high reulceration rates. To change this pattern of behavior based on a misconception, we need to start with how we as clinicians are conceptualizing treatment with offloading devices.

Non-removable offloading devices as commitment devices: Commitment devices are voluntary restrictions people put on their future selves to resist short-term temptations and achieve long-term goals. In this paper, it is suggested that a change from viewing non-removable offloading devices as means to force compliance, to viewing them as commitment devices could facilitate a change to a clinical thinking that emphasizes the importance of high adherence without compromising respect for patient autonomy.

Conclusion: Viewing non-removable offloading devices as commitment devices seems to be a promising approach to emphasize the importance of adherence while respecting patient autonomy. Hopefully, patients' higher appreciation of the role of adherence can lead to higher adherence to using therapeutic footwear after healing and consequently to reduced reulceration rates.

Keywords: Diabetes complications, Diabetic foot, Shoes, Patient compliance, Treatment adherence and compliance, Casts, surgical, Shoes, Orthotic devices
\end{abstract}

\section{Background}

Diabetic foot ulcers are a common and devastating complication of diabetes, and are associated with significant morbidity, mortality, and risk of amputation [1]. Nonremovable offloading devices are recommended in the treatment of uncomplicated plantar foot ulcers [2] because adherence to using removable offloading devices is often low [3, 4]. Once the ulcer is healed, patients change to using removable offloading devices, typically therapeutic footwear, to mitigate plantar pressures and prevent reulceration [5]. One may think that the experience of effective healing when using a non-removable device would convince patients of the importance of

Correspondence: gustav.jarl@regionorebrolan.se

${ }^{1}$ Department of Prosthetics and Orthotics, Faculty of Medicine and Health, Örebro University, SE 70182 Örebro, Sweden

${ }^{2}$ University Health Care Research Center, Faculty of Medicine and Health, Örebro University, SE 70182 Örebro, Sweden adherence, resulting in high adherence to wearing therapeutic footwear after healing and thus ensuring low reulceration rates. Unfortunately, many patients do not seem to acknowledge that the non-removability of the device ensures high adherence to using it, which allows the ulcer to heal; instead, they attribute the entire healing outcome to the device's offloading effect, that is, the ability of the device to reduce mechanical stresses on the ulcer. In one study [6], patients were interviewed about their experiences of using total contact casts and removable walkers. Interestingly, they were aware of the more effective ulcer healing when using total contact casts but attributed it - falsely - to casts offloading the ulcer more effectively than walkers do; they did not attribute better healing to the non-removability of casts resulting in higher adherence. This stands in stark contrast to research results, which demonstrate that walkers can offload forefoot ulcers equally effectively as casts $[7,8]$

(c) The Author(s). 2019 Open Access This article is distributed under the terms of the Creative Commons Attribution 4.0 International License (http://creativecommons.org/licenses/by/4.0/), which permits unrestricted use, distribution, and 
and that walkers provide similar healing outcomes as casts if the walkers are rendered non-removable [6]. Patients' underestimation of the importance of adherence may be one of the reasons for low adherence to wearing therapeutic footwear after ulcer healing [9] and for the high reulceration rates: approximately $40 \%$ of patients develop a new ulcer within the first year after healing [1].

One may speculate that the way clinicians frame and present treatment with non-removable offloading devices may not communicate to patients the importance of high adherence. All too often, the clinical focus is on the device itself, leaving patients with the impression that it is the device per se that heals the ulcer. In reality, healing of uncomplicated plantar ulcers is mainly determined by two factors combined: effective offloading of the ulcer and high adherence to using the offloading device. Hence, non-removable devices have been proposed as a way to force compliance and thereby reach the desired level of adherence [4, 10]. However, the concept of non-removable devices as a means to force compliance has a paternalistic connotation which is not compatible with viewing patients as partners in decision-making. Furthermore, it conveys the picture of clinicians as active and patients as passive in the decision. Although unintended, these connotations may counteract the sense of long-term personal responsibility for adherence that is crucial after healing, when removable devices are used to prevent reulceration. Thus, an alternative way to conceptualize treatment with nonremovable offloading devices is needed.

\section{Non-removable offloading devices as commitment devices}

Commitment devices are voluntary restrictions that people put on their future selves to resist short-term temptations and achieve long-term goals [11]. For example, a person may undergo gastric bypass surgery to guard against future temptations to overeat, and thereby reduce calorie intake and lose weight. This line of thinking fits well in the context of adherence to using offloading devices; the long-term goal is to heal a foot ulcer and the short-term temptation is to engage in "strategic non-adherence", that is, purposely being non-adherent in an attempt to live a normal life $[12,13]$.The solution is to use a non-removable device, which in this sense could be labeled a commitment device; the patient agrees to use the device and commits to using it continuously, even when his or her future self is tempted to remove it. In contrast to viewing non-removable devices as a means to force compliance on a passive patient, the patient's current self can be invited to take an active decision to restrict the opportunities for his or her future self to be non-adherent (Fig. 1).

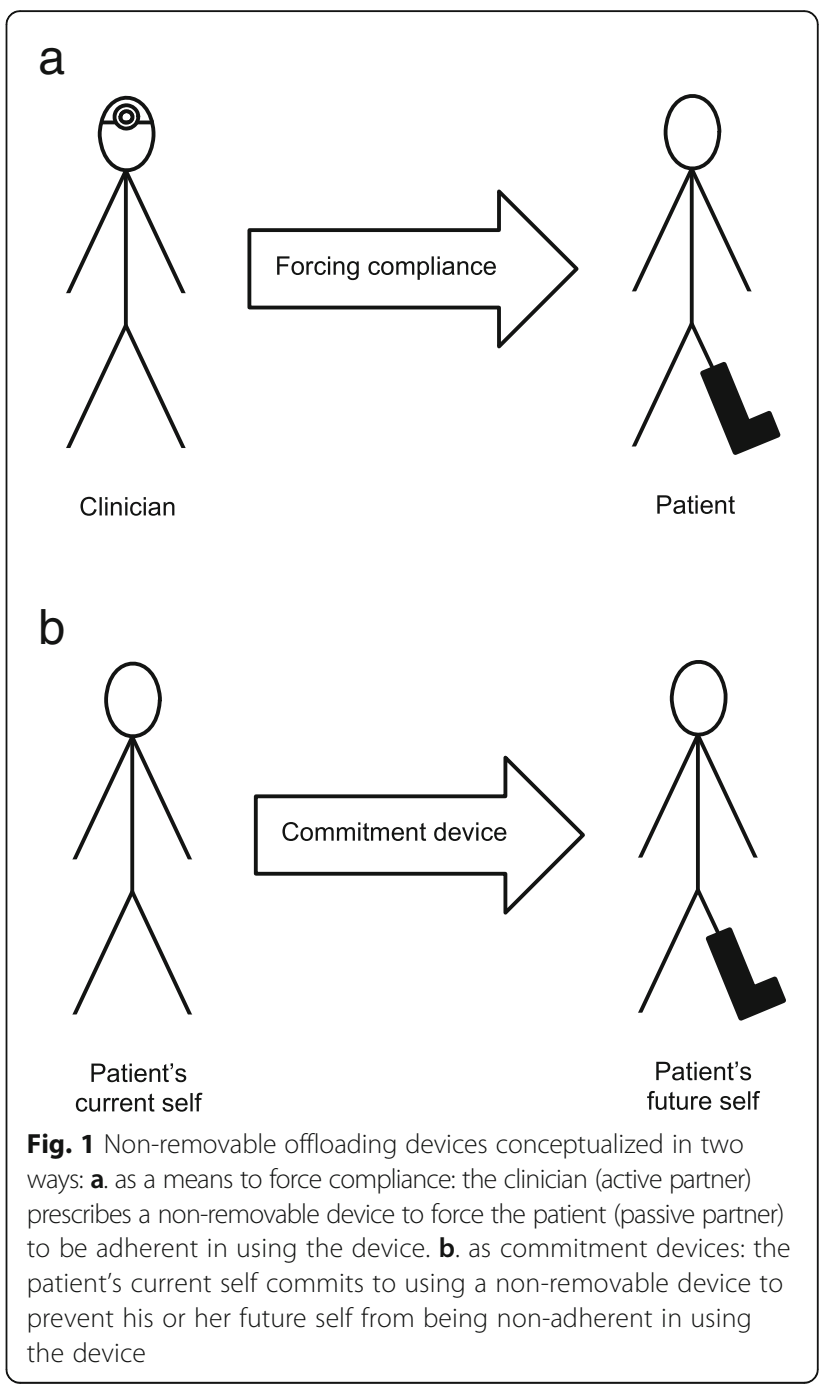

Hence, the importance of adherence is made salient without compromising respect for the patient's autonomy and self-determination. In addition, conceptualizing non-removable devices as commitment devices highlights the need to discuss the gap between intentions and future behaviors with patients [11], a discussion that should precede the transition from using a non-removable device during treatment to using removable offloading devices after healing. Now the focus is on educating the patient about the effectiveness of the offloading device, typically therapeutic footwear, and establishing long-term habits of high adherence. The literature suggests different approaches to strengthen the patient's motivation for adherence and to bridge the intention-action gap, but the evidence is in most cases weak and more research is needed. For example, patients may be educated about the offloading effect by measuring and visualizing plantar pressures [14], and adherence may be improved with motivational interviewing [15] and other structured communication techniques, such as person-centered 
communication and shared decision-making [16]. Furthermore, patients can be advised to keep their therapeutic footwear visible at home to provide a cue to use it and to put their conventional shoes away to eliminate the temptation to use them [17]. Hopefully, by emphasizing the importance of adherence, ensuring that the patient is an active agent during ulcer treatment, and addressing the intention-action gap, higher adherence to removable offloading devices after healing can be achieved.

Although commitment devices per se have not been discussed previously in the context of offloading diabetic foot ulcers, the underlying principle of self-imposed restrictions is already at work in the field. A study in which people with foot ulcers wore non-removable therapeutic footwear illustrates this [18]. In the questionnaire asking participants for perceived advantages and disadvantages with the treatment regimen, one participant reported that an advantage of non-removable footwear was that he was not tempted to walk without his therapeutic footwear. This illustrates the idea of viewing non-removable offloading devices as commitment devices; the idea is not to force compliance on patients but to suggest an effective, still voluntary, means to improve adherence through a self-imposed restriction on everyday choices available to the person's future self.

How we choose to name things influences how we perceive them and, by extension, how we act in clinical practice. Thus, it is important to choose and use concepts that provide an appropriate clinical mindset when meeting our patients. For example, in one study, the term "diabetic foot attack" was introduced to emphasize the urgency of certain clinical presentations of diabetic foot disease, such as an acutely inflamed foot with rapidly progressing tissue necrosis [19]. Another study proposed the "in remission" concept as an alternative to viewing patients as being cured, in order to prepare patients and clinicians for inevitable future complications and to emphasize the need for frequent follow-up [20]. A third study suggested the "process perspective" to highlight diabetic foot disease as a single process consisting of both active (healing) and latent (prevention) phases, which could be a more fruitful model for understanding inadequate patient behaviors that are not easily understood from a dichotomous healing-prevention perspective [21]. Hopefully, conceptualizing non-removable offloading devices as commitment devices to aid adherence could be a part of this expanding conceptual framework to support clinicians in their work and their communication with patients.

\section{Conclusions}

Viewing non-removable offloading devices as commitment devices seems to be a promising approach to emphasize the importance of adherence without compromising respect for patient autonomy. Hopefully, this will result in higher adherence to using removable offloading devices after healing, which would in turn lower reulceration rates. Other researchers are invited to elaborate on this concept and investigate its practical implications.

\section{Acknowledgements \\ Not applicable.}

Authors' contributions

The sole author did all the work on this manuscript. The author read and approved the final manuscript.

Funding

Not applicable.

Availability of data and materials

Not applicable.

Ethics approval and consent to participate

Not applicable.

Consent for publication

Not applicable.

\section{Competing interests}

GJ is a consultant for Novo Nordisk but does not consider this to be a relevant conflict of interest in this work.

Received: 12 June 2019 Accepted: 9 August 2019

Published online: 19 August 2019

\section{References}

1. Armstrong DG, Boulton AJM, Bus SA. Diabetic foot ulcers and their recurrence. N Engl J Med. 2017;376:2367-75.

2. Bus SA, Armstrong DG, van Deursen RW, Lewis JE, Caravaggi CF, Cavanagh PR. IWGDF guidance on footwear and offloading interventions to prevent and heal foot ulcers in patients with diabetes. Diabetes Metab Res Rev. 2016;32(Suppl 1):25-36.

3. Armstrong DG, Lavery LA, Kimbriel HR, Nixon BP, Boulton AJ. Activity patterns of patients with diabetic foot ulceration: patients with active ulceration may not adhere to a standard pressure off-loading regimen. Diabetes Care. 2003;26:2595-7.

4. Ha Van G, Siney H, Hartmann-Heurtier A, Jacqueminet S, Greau F, Grimaldi A. Nonremovable, windowed, fiberglass cast boot in the treatment of diabetic plantar ulcers: efficacy, safety, and compliance. Diabetes Care. 2003;26:2848-52.

5. Bus SA, Van Netten JJ, Lavery LA, Monteiro-Soares M, Rasmussen A, Jubiz Y, Price PE. IWGDF guidance on the prevention of foot ulcers in at-risk patients with diabetes. Diabetes Metab Res Rev. 2016;32:16-24.

6. Health Quality Ontario. Fibreglass total contact casting, removable cast walkers, and irremovable cast walkers to treat diabetic neuropathic foot ulcers: a health technology assessment. Ont Health Technol Assess Ser. 2017;17:1-124.

7. Gutekunst DJ, Hastings MK, Bohnert KL, Strube MJ, Sinacore DR. Removable cast walker boots yield greater forefoot off-loading than total contact casts. Clin Biomech (Bristol, Avon). 2011;26:649-54.

8. Lavery LA, Vela SA, Lavery DC, Quebedeaux TL. Reducing dynamic foot pressures in high-risk diabetic subjects with foot ulcerations. A comparison of treatments. Diabetes Care. 1996:19:818-21.

9. Waaijman R, Keukenkamp R, de Haart M, Polomski WP, Nollet F, Bus SA. Adherence to wearing prescription custom-made footwear in patients with diabetes at high risk for plantar foot ulceration. Diabetes Care. 2013;36: 1613-8

10. Armstrong DG, Nguyen HC, Lavery LA, van Schie $\mathrm{CH}$, Boulton AJ, Harkless LB. Off-loading the diabetic foot wound: a randomized clinical trial. Diabetes Care. 2001;24:1019-22. 
11. Rogers T, Milkman KL, Volpp KG. Commitment devices: using initiatives to change behavior. JAMA. 2014;311:2065-6.

12. Beattie AM, Campbell R, Vedhara K. What ever I do it's a lost cause.' the emotional and behavioural experiences of individuals who are ulcer free living with the threat of developing further diabetic foot ulcers: a qualitative interview study. Health Expect. 2014;17:429-39.

13. Coffey L, Mahon C, Gallagher P. Perceptions and experiences of diabetic foot ulceration and foot care in people with diabetes: a qualitative metasynthesis. Int Wound J. 2018:1-28.

14. Mueller MJ, Smith KE, Commean PK, Robertson DD, Johnson JE. Use of computed tomography and plantar pressure measurement for management of neuropathic ulcers in patients with diabetes. Phys Ther. 1999;79:296-307.

15. Keukenkamp R, Merkx MJ, Busch-Westbroek TE, Bus SA. An explorative study on the efficacy and feasibility of the use of motivational interviewing to improve footwear adherence in persons with diabetes at high-risk of foot ulceration. J Am Podiatr Med Assoc. 2018;108:90-9.

16. Van Netten JJ, Francis A, Morphet A, Fortington LV, Postema K, Williams A. Communication techniques for improved acceptance and adherence with therapeutic footwear. Prosth Orthot Int. 2017:41:201-4.

17. Jarl G, Alnemo J, Tranberg R, Lundqvist L-O. Predictors of adherence to using therapeutic shoes among people with diabetic foot complications. In: 8th international symposium on the diabetic foot. The Hague; 2019.

18. Jarl G, Tranberg R. An innovative sealed shoe to off-load and heal diabetic forefoot ulcers - a feasibility study. Diabetic Foot \& Ankle. 2017;8:1348178.

19. Vas PR, Edmonds M, Kavarthapu V, Rashid H, Ahluwalia R, Pankhurst C Papanas N. The diabetic foot attack:"'tis too late to retreat!". Int J Low Extrem Wounds. 2018;17(1):7-13.

20. Armstrong DG, Mills JL. Toward a change in syntax in diabetic foot care: prevention equals remission. J Am Podiatr Med Assoc. 2013;103:161-2.

21. Jarl G, Lundqvist L-O. Beyond dichotomous thinking: a process perspective on diabetic foot disease. Diabetic Foot \& Ankle. 2017;8:1380477.

\section{Publisher's Note}

Springer Nature remains neutral with regard to jurisdictional claims in published maps and institutional affiliations.

Ready to submit your research? Choose BMC and benefit from:

- fast, convenient online submission

- thorough peer review by experienced researchers in your field

- rapid publication on acceptance

- support for research data, including large and complex data types

- gold Open Access which fosters wider collaboration and increased citations

- maximum visibility for your research: over $100 \mathrm{M}$ website views per year

At $\mathrm{BMC}$, research is always in progress.

Learn more biomedcentral.com/submissions 